\title{
A rare case of Castleman's disease presenting as a pulmonary mass mimicking pulmonary malignancy
}

\author{
Bashyal $\mathrm{R}^{1}$, Lee $\mathrm{MC}^{1}$, Thakur B ${ }^{2}$, Singh $\mathrm{LN}^{3}$ \\ ${ }^{1}$ Department of Pathology, BP Koirala Memorial Cancer Hospital, Bharatpur, Nepal \\ ${ }^{2}$ Department of Surgery, BP Koirala Memorial Cancer Hospital, Bharatpur, Nepal \\ ${ }^{3}$ Department of Radiology, BP Koirala Memorial Cancer Hospital, Bharatpur, Nepal
}

\author{
Keywords: \\ Castleman disease; \\ Pulmonary malignancy; \\ IL-6; \\ HHV-8; \\ Kaposi sarcoma
}

\begin{abstract}
Castleman disease is a rare disorder of the lymphoid system characterized by noncancerous growths that may develop in lymph node tissues throughout the body. Most often this occurs in the neck, mediastinum, and abdomen where lymph nodes aggregate. The etiology is thought to be due to antigenic hyperstimulation of unknown origin. Two histological subtypes are described with different clinical presentations and therapeutic implications. Diagnosis is frequently accomplished only by histological analysis after surgery since no specific features have been found in imaging studies. Surgical excision is both diagnostic and curative in localized forms, whereas additional therapies are required in multicentric forms. Here we describe a case of castleman disease adjacent to the right pulmonary hilum loosely adherent to the interlobar branch of the right pulmonary artery that mimicked tuberculosis or malignancy in a 30-year-old man who underwent curative surgical removal of the mass.
\end{abstract}

\section{INTRODUCTION}

Castleman's disease (CD) is a rare atypical lymphoproliferative disorder whose morphology, soon after the original presentation of Castleman et al, has been definitely subdivided into hyaline vascular (HV), plasma cell (PC) and mixed variants. The former occurs much more frequently than the latter and is usually localized to the mediastinum or pulmonary hilum. The latter involves lymph nodes separately or in aggregations and often displays multicentricity with systemic symptoms including autoimmune phenomena and aggressive course.

\section{Correspondence:}

Dr. Rita Bshyal, $M D$

Department of Pathology, BP Koirala Memorial Cancer Hospital Bharatpur, Nepal.
Small hyalinized and hypervascular germinal centers with hypervascular interfollicular stroma and sinus effacement are common features of the $\mathrm{HV}$ variant. Hyperplastic germinal centers with plasma cell aggregates in lymph node paracortex and partially spared sinuses are characteristic features of the PC variant. The frequent concomitance of the $\mathrm{HV}$ and PC types at separate sites, together with transient morphological patterns from one type to the other and from the localized to multicentric form during the course of the disease, along with B and T cell impaired functions, with frequent development of autoantibodies, have suggested that $\mathrm{CD}$ is a single disorder related to immune dysregulation. A key event in the pathogenesis of $\mathrm{CD}$ has been recently suggested to be an abnormal production of a B cell growth factor, such as Interleukin-6 (IL-6), leading to lymphoproliferation and plasma cell differentiation and 


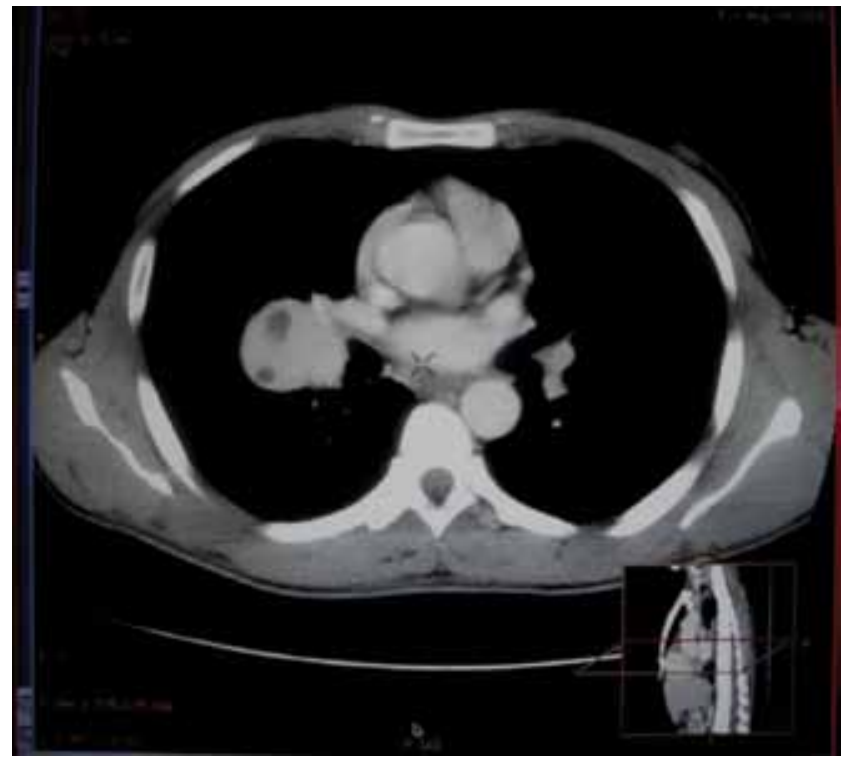

Figure 1: CT scan of chest shows well defined, smoothly marginated, solid mass in right hilum which show strong contrast enhancement with small non-enhancing areas

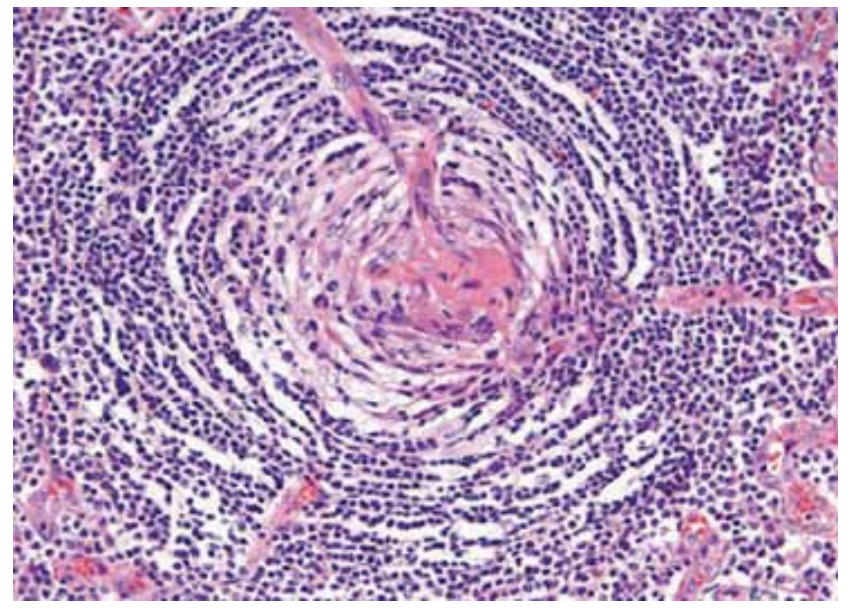

Figure 2A: Atrophic follicle containing radiating blood vessel simulating a lollypop appearance and surrounding mantle lymphocytes forming a ring imparting an onion-skin appearance (HE stain, X40).

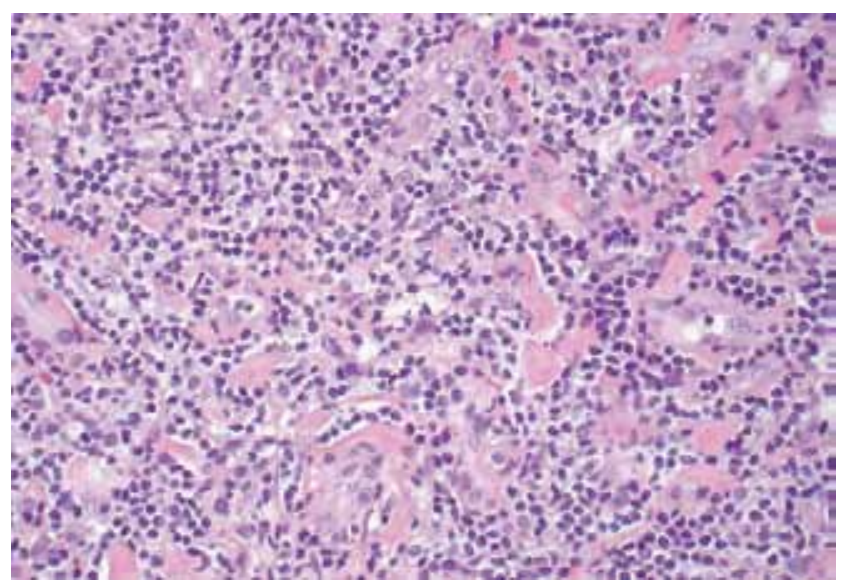

Figure 2B: Interfollicular areas consisting of numerous hyalinized vessels with enlarged endothelial cells (HE stain, X40) being involved in the oncogenesis of plasmacytoma. In this event, Kaposi's sarcoma associated virus (HHV-8), which has been found in many cases of CD, especially in the multicentric form, could play a crucial role both in producing IL-6 and releasing angiogenic factors. A possible differentiation block may lead to the development of a malignant lymphoma. Kaposi's sarcoma or other malignant neoplasias can be consequences of the immunodeficiency typical of CD. An association of CD with thymic carcinoma and lung carcinoma are reported.

\section{REPORT}

A 30 year old male wood worker who was a chronic alcoholic and tobacco chewer came with complaints of right sided chest pain and an intermittent cough for 1 month. Physical examination of the patient revealed good general condition. The vitals were within the normal limits. The remainder of systemic examination was normal.

The laboratory findings were significant for following values: WBC count 8400/cumm (neutrophils-71, lymphocytes-25, eosinophils-01 and monocytes-03), haemoglobin-13.0gm\%. The remaining biochemical evaluation values and the coagulation profile were within normal limits. Human immunodeficiency virus and hepatitis virus (B and $\mathrm{C}$ ) titers were not indicative of any recent or remote infections.

Bronchoscopy revealed normal segmental bronchi and Ultrasonogram (USG) abdomen revealed a normal scan. The chest radiograph was normal. The computerized tomography (CT) of chest revealed well-defined, smoothly marginated, solid mass $(4.4 \times 4 \times 3.6 \mathrm{~cm})$ in the right hilum which showed strong contrast enhancement with small nonenhancing areas (fig 1).

Grossly, the mass was $4 \times 3.5 \times 3 \mathrm{~cm}$, single; cut surface was homogenous and grey-white coloured. Histopathologic examination revealed a mass of lymphoid tissue having germinal center follicles with prominent mantle zones comprised of small, mature lymphocytes arranged in concentric layers with prominent hyalinized vessels. Prominent endothelial cells were seen in the vessels extending into the adjacent interfollicular area (fig. 2 $\mathrm{A}$ and B). Morphologic features showed no abnormal haematolymphoid population and was labelled as hyaline vascular type of Castlemans disease. The post operative period was uneventful.

\section{DISCUSSION}

$\mathrm{CD}$ is a benign lymphoproliferative disorder characterized by enlarged hyperplastic lymph nodes. In 1956 Benjamin Castleman and his associates described this disease. ${ }^{1}$ Since then it is also recognized as angiofollicular lymphoid hyperplasia, giant lymph node hyperplasia, lymphoid hyperplasia, benign lymphoma, and follicular lympho- 
reticuloma. ${ }^{2}$ It can present as a localized mass or as a multicentric disorder. Its presents as a pulmonary hilar mass loosely adherent to the pulmonary artery has been reported in the literature. ${ }^{3}$ Histologically, three variants are recognized. They are HV, PC, and intermediate type. ${ }^{4} \mathrm{HV}$ type is the most common presentation. This type is usually localized to the mediastinum or pulmonary hilum. ${ }^{5}$ This is similar to our patient who presented with mass in the pulmonary hilum. Lymph nodes from various animal models and patients with CD implicate IL-6 as a causative agent for the commonly observed systemic manifestations..$^{5-10}$ Localized disease usually has a benign course and can present as an asymptomatic mass or with compression effect in respective area and also as pyrexia of unknown origin, weight loss or anemia. ${ }^{5}$ Multicentric disease is usually a systemic illness involving the retroperitoneum, neck, parotids and muscles. It can also present as a disseminated lymphadenopathy. It is aggressive and usually the fatal course is associated with infectious complications and risks for malignant tumors such as lymphoma or Kaposi sarcoma.

The classical histopathological picture consists of thickened hyalinized capillaries within the follicle centres. There is perifollicular vascular proliferation with concentric layering of cells within germinal centres. There may be more than one germinal center within a single follicle.

Surgical excision is curative for the localized disease. Multicentric disease is usually associated with systemic manifestations and its prognosis is guarded. Its management includes a multimodality approach comprising of surgery, combination chemotherapy, steroids and radiation. Lately, anti-IL-6 receptor antibody therapy has been tried. ${ }^{11}$ Adjunctive radiotherapy could be an option in patients who are at high risk of recurrence or unsuitable for surgery. ${ }^{12}$

\section{CONCLUSION}

$\mathrm{CD}$ is a challenging histopathological case to evaluate because of its rare incidence and nonspecific clinical features and results of radiological examination, and definite diagnosis depends on true histology.

\section{REFERENCES}

1. Castleman B, Iverson L, Menendez VP. Localized mediastinal lymphonode hyperplasia resembling thymoma. Cancer 1956;9:82230.

2. Herrada J. Cabanillas F, Rice L, Mannins J, Pugh W. The clinical behavior of localized and multicenteric Castleman's disease. Ann Intern Med 1998;128:657-62.

3. Wang SH, Ruan Z, Huang HL, Song KS. A rare case of Castleman disease presenting as pulmonary mass mimicking central pulmonary malignancy. Chin Med J 2009;122:990-1.

4. Halkic N. Cornu P, Mosimann F. Castleman's disease: 2 unusual cases. Schweiz Med Wochenschr 1998;128:331-6.

5. Palestro G, Turrini F, Pagano M, Chiusa L. Castleman's disease. Adv Clin Pathol 1999;3:11-22.

6. Hsu SM, Waldron JA, Xie SS, Barlogie B. Expression of interleukin-6 in Castleman's disease. Hum Pathol 1993;24:833-9.

7. Lotz M. Interleukin-6. Cancer Invest 1993;11:732-42.

8. Yoshizaki $\mathrm{K}$, Matsuda T, Nishimoto $\mathrm{N}$ et al. Pathogenic significance of interleukin-6 (IL-6/BSF-2) in Castleman's disease. Blood 1989;74:1360-7.

9. Leger-Ravet MB, Peuchmaur M, Devergne O et al. Interleukin-6 gene expression in Castleman's disease. Blood 1991;78:2923-30.

10. Brandt SJ, Bodine DM, Dunbar CE, Nienhuis AW. Dysregulated interleukin-6 expression produces a syndrome resembling Castleman's disease in mice. J Clin Invest 1990;86:592-9.

11. Nishimoto N, Sasai M, Shima Y et al. Improvement in Castleman's disease by humanised anti- interkeukin-6 receptor antibody therapy. Blood 2000;95:56-61.

12. Fisher ER, Sieracki JC, Goldenberg DM. Identity and nature of isolated lymphoid tumors (so-called nodal hyperplasia, hamartoma, and angiomatous hamartoma) as revealed by histologic, electron microscopic, and heterotransplantation studies. Cancer 1970;25:1286-300 